\title{
Cohomology of the variational complex in BRST theory
}

\author{
G. SARDANAShVILY \\ Department of Theoretical Physics, Moscow State University, 117234 Moscow, Russia \\ E-mail: sard@grav.phys.msu.su
}

\begin{abstract}
We show that cohomology of the variational complex in the field-antifield BRST theory on an arbitrary manifold is equal to the de Rham cohomology of this manifold.
\end{abstract}

\section{Introduction}

In the field-antifield BRST theory, the antibracket and the BRST operator are defined by means of the variational operator (see, e.g., [17]). To introduce this variational operator in a rigorous algebraic way, one can replace the calculus in functionals with the calculus in jets of fields and antifields, and can construct the variational complex [4, 0, 11]. Furthermore, one has proved that the variational complex in BRST theory on a contractible manifold $\mathbb{R}^{n}$ is exact [10, 11, 13]. This means that the kernel of the variational operator $\delta$ coincides with the image of the horizontal (or total) differential $d_{H}$. Therefore, main objects in the field-antifield BRST theory on $\mathbb{R}^{n}$ are defined modulo $d_{H^{-}}$exact forms. Let us mention, e.g., the local BRST cohomology.

Here, the variational complex in the field-antifield BRST theory on an arbitrary smooth manifold $X$ is studied; that requires a (global) differential geometric definition of ghosts, antifields, and their jets. We show that cohomology of this variational complex equals the de Rham cohomology of $X$. In other words, the obstruction to the exactness of the variational complex in BRST theory lies only in closed non-exact forms on $X$. This fact enables one to generalize many constructions of the field-antifield BRST theory on $\mathbb{R}^{n}$ to that on an arbitrary manifold $X$. In particular, global descent equations on $X$ can be defined [16].

For the sake of simplicity, we will consider the case of even physical fields and even irreducible gauge transformations with a finite number of generators. Then ghosts are odd, and antifields are odd and even. For instance, this is the case of the Yang-Mills theory. One says that physical fields, ghosts and antifields constitute a physical basis.

We start from cohomology of the variational complex of even classical fields. Cohomology of the variational complex in BRST theory is studied in a similar way. 


\section{The variational complex of classical fields}

In classical field theory, fields are represented by sections of a smooth fibre bundle $Y \rightarrow X$. Put further $\operatorname{dim} X=n$.

Remark. Smooth manifolds throughout are real, finite-dimensional, Hausdorff, secondcountable (i.e., paracompact), and connected. The standard notation of jet formalism is utilized (see, e.g., [14, 22]). We follow the terminology of [12, 18], where a sheaf $S$ is a particular topological bundle and $\Gamma(S)$ denotes the group of global sections of $S$.

The configuration space of Lagrangian formalism on a fibre bundle $Y \rightarrow X$ is the infinite order jet space $J^{\infty} Y$ of $Y \rightarrow X$. It is defined as a projective limit of the inverse system

$$
X \stackrel{\pi}{\longleftarrow} Y \stackrel{\pi_{0}^{1}}{\longleftarrow} \cdots J^{r-1} Y \stackrel{\pi_{r-1}^{r}}{\longleftarrow} J^{r} Y \longleftarrow \cdots
$$

of finite order jet manifolds $J^{r} Y$ of $Y \rightarrow X$, where $\pi_{r-1}^{r}$ are affine bundles. One can say that $J^{\infty} Y$ consists of the equivalence classes of sections of $Y \rightarrow X$ identified by their Taylor series at points of $X$. Endowed with the projective limit topology, the ionfinite order jet space $J^{\infty} Y$ is a paracompact Fréchet manifold [27]. A bundle coordinate atlas $\left\{U_{Y},\left(x^{\lambda}, y^{i}\right)\right\}$ of $Y \rightarrow X$ yields the manifold coordinate atlas

$$
\left\{\left(\pi_{0}^{\infty}\right)^{-1}\left(U_{Y}\right),\left(x^{\lambda}, y_{\Lambda}^{i}\right)\right\}, \quad 0 \leq|\Lambda|
$$

of $J^{\infty} Y$, together with the transition functions

$$
y_{\lambda+\Lambda}^{\prime i}=\frac{\partial x^{\mu}}{\partial x^{\prime \lambda}} d_{\mu} y_{\Lambda}^{\prime i}
$$

where $\Lambda=\left(\lambda_{k} \ldots \lambda_{1}\right), \lambda+\Lambda=\left(\lambda \lambda_{k} \ldots \lambda_{1}\right)$ are multi-indices and

$$
d_{\lambda}=\partial_{\lambda}+\sum_{|\Lambda| \geq 0} y_{\lambda+\Lambda}^{i} \partial_{i}^{\Lambda}
$$

is the total derivative.

Let us introduce the differential calculus on $J^{\infty} Y$. With the inverse system (11), one has the direct system

$$
\mathcal{O}^{*}(X) \stackrel{\pi^{*}}{\longrightarrow} \mathcal{O}_{0}^{*} \stackrel{\pi_{0}^{1 *}}{\longrightarrow} \mathcal{O}_{1}^{*} \stackrel{\pi_{1}^{2 *}}{\longrightarrow} \cdots \mathcal{O}_{r}^{*} \stackrel{\pi_{r}^{r+1 *}}{\longrightarrow} \cdots
$$

of differential algebras $\mathcal{O}_{r}^{*}$ of exterior forms on finite order jet manifolds $J^{r} Y$, where $\pi_{r-1}^{r *}$ are the pull-back monomorphisms. The direct limit of this direct system is the differential 
algebra $\mathcal{O}_{\infty}^{*}$ which consists of all exterior forms on finite order jet manifolds modulo the pull-back identification. In particular, $\mathcal{O}_{\infty}^{*}$ is the ring of the pull-back onto $J^{\infty} Y$ of smooth real functions on finite order jet manifolds.

For short, we agree to call elements of $\mathcal{O}_{\infty}^{*}$ the exterior forms on $J^{\infty} Y$. Of course, these forms are of bounded jet order. Restricted to a coordinate chart $\left(\pi_{0}^{\infty}\right)^{-1}\left(U_{Y}\right)$ of $J^{\infty} Y$, they can be written in a coordinate form, where horizontal forms $\left\{d x^{\lambda}\right\}$ and contact 1forms $\left\{\theta_{\Lambda}^{i}=d y_{\Lambda}^{i}-y_{\lambda+\Lambda}^{i} d x^{\lambda}\right\}$, together with the constant function $\mathbf{1}$, constitute the set of generators of the $\mathcal{O}_{\infty}^{0}$-algebra $\mathcal{O}_{\infty}^{*}$. There is the canonical splitting

$$
\mathcal{O}_{\infty}^{*}=\underset{k, s}{\oplus} \mathcal{O}_{\infty}^{k, s}, \quad 0 \leq k, \quad 0 \leq s \leq n,
$$

of $\mathcal{O}_{\infty}^{*}$ into $\mathcal{O}_{\infty}^{0}$-modules $\mathcal{O}_{\infty}^{k, s}$ of $k$-contact and $s$-horizontal forms, together with the corresponding projections

$$
\begin{aligned}
& h_{k}: \mathcal{O}_{\infty}^{*} \rightarrow \mathcal{O}_{\infty}^{k, *}, \quad 0 \leq k, \\
& h^{s}: \mathcal{O}_{\infty}^{*} \rightarrow \mathcal{O}_{\infty}^{*, s}, \quad 0 \leq s \leq n .
\end{aligned}
$$

Accordingly, the exterior differential on $\mathcal{O}_{\infty}^{*}$ is decomposed into the sum $d=d_{H}+d_{V}$ of horizontal and vertical differentials

$$
\begin{array}{ll}
d_{H} \circ h_{k}=h_{k} \circ d \circ h_{k}, & d_{H}(\phi)=d x^{\lambda} \wedge d_{\lambda}(\phi), \\
d_{V} \circ h^{s}=h^{s} \circ d \circ h^{s}, & d_{V}(\phi)=\theta_{\Lambda}^{i} \wedge \partial_{i}^{\Lambda} \phi, \quad \phi \in \mathcal{O}_{\infty}^{*} .
\end{array}
$$

Lagrangians, Euler-Lagrange operators, and other objects of a familiar Lagrangian field theory are elements of the differential algebra $\mathcal{O}_{\infty}^{*}$. They can be introduced in an algebraic way by constructing the variational complex of the algebra $\mathcal{O}_{\infty}^{*}$.

The $\mathbb{R}$-module endomorphism

$$
\begin{aligned}
& \tau=\sum_{k>0} \frac{1}{k} \bar{\tau} \circ h_{k} \circ h^{n}, \\
& \left.\bar{\tau}(\phi)=(-1)^{|\Lambda|} \theta^{i} \wedge\left[d_{\Lambda}\left(\partial_{i}^{\Lambda}\right\rfloor \phi\right)\right], \quad 0 \leq|\Lambda|, \quad \phi \in \mathcal{O}_{\infty}^{>0, n},
\end{aligned}
$$

of $\mathcal{O}_{\infty}^{*}$ is defined (see, e.g., [8, 14, 29]). It is a projector, i.e., $\tau \circ \tau=\tau$, and obeys the relations

$$
\tau \circ d_{H}=0, \quad \tau \circ d \circ \tau-\tau \circ d=0 .
$$

Put $E_{k}=\tau\left(\mathcal{O}_{\infty}^{k, n}\right)$. The variational operator on $\mathcal{O}_{\infty}^{*, n}$ is defined as the morphism $\delta=\tau \circ d$. It is nilpotent, and has the property

$$
\delta \circ \tau-\tau \circ d=0 .
$$


Since the operators $d_{H}$ and $\delta$ are nilpotent, and the relations (3) hold, we have the complex

$$
0 \rightarrow \mathbb{R} \rightarrow \mathcal{O}_{\infty}^{0} \stackrel{d_{H}}{\longrightarrow} \mathcal{O}_{\infty}^{0,1} \stackrel{d_{H}}{\longrightarrow} \cdots \stackrel{d_{H}}{\longrightarrow} \mathcal{O}_{\infty}^{0, n} \stackrel{\delta}{\longrightarrow} E_{1} \stackrel{\delta}{\longrightarrow} E_{2} \longrightarrow \cdots
$$

called the variational complex. Elements of its term $\mathcal{O}_{\infty}^{0, n}$ are Lagrangians, while those of $E_{1}$ are Euler-Lagrange operators. There are the well-known statements summarized usually as the algebraic Poincaré lemma (see, e.g., [23, 29]).

Lemma 1. If $Y$ is a contractible fibre bundle $\mathbb{R}^{n+m} \rightarrow \mathbb{R}^{n}$, the variational complex (5) is exact.

To obtain cohomology of the variational complex (5) in the case of an arbitrary smooth fibre bundle $Y \rightarrow X$, let us enlarge the differential algebra $\mathcal{O}_{\infty}^{*}$ as follows 15, 16.

Let $\mathfrak{T}_{r}^{*}$ be the sheaf of germs of exterior forms on $J^{\infty} Y$ and $\Gamma\left(\mathfrak{T}_{\infty}^{*}\right)$ the differential algebra of its global sections. One can say that the algebra $\Gamma\left(\mathfrak{T}_{\infty}^{*}\right)$ consists of exterior forms on $J^{\infty} Y$ which coincide locally (i.e., around each point of $J^{\infty} Y$ ) with the pull-back of exterior forms on finite-order jet manifolds. In particular, $\Gamma\left(\mathfrak{T}_{\infty}^{0}\right)$ is the ring of real functions on $J^{\infty} Y$ such that, given $f \in \Gamma\left(\mathfrak{T}_{\infty}^{0}\right)$ and any point $q \in J^{\infty} Y$, there exists a neighborhood of $q$ where $f$ coincides with the pull-back of a smooth function on some finite order jet manifold. There is the natural monomorphism $\mathcal{O}_{\infty}^{*} \rightarrow \Gamma\left(\mathfrak{T}_{\infty}^{*}\right)$.

Note that, in comparison with $\mathcal{O}_{\infty}^{0}$, the jet order of elements of $\Gamma\left(\mathfrak{T}_{\infty}^{0}\right)$ need not be bounded. Therefore, the algebra $\Gamma\left(\mathfrak{T}_{\infty}^{0}\right)$ has a limited physical application. We involve it because the paracompact space $J^{\infty} Y$ admits a partition of unity by elements of the ring $\Gamma\left(\mathfrak{T}_{\infty}^{0}\right)$ [27. It follows that the sheaves of $\Gamma\left(\mathfrak{T}_{\infty}^{0}\right)$-modules on $J^{\infty} Y$ are fine and, consequently, acyclic. Therefore, the abstract de Rham theorem 18 can be called into play in order to obtain cohomology of the differential algebra $\Gamma\left(\mathfrak{T}_{\infty}^{*}\right)$. Then one proves that the algebras $\mathcal{O}_{\infty}^{*}$ and $\Gamma\left(\mathfrak{T}_{\infty}^{*}\right)$ have the same cohomology.

Since $\tau$ and $\delta$ on $\mathcal{O}_{\infty}^{*}$ are pointwise operators, their direct limits are defined on the sheaf $\mathfrak{T}_{\infty}^{*}$ and possess the properties (3) and (4). Then we have the variational complex of sheaves

$$
0 \rightarrow \mathbb{R} \rightarrow \mathfrak{T}_{\infty}^{0} \stackrel{d_{H}}{\longrightarrow} \mathfrak{T}_{\infty}^{0,1} \stackrel{d_{H}}{\longrightarrow} \cdots \stackrel{d_{H}}{\longrightarrow} \mathfrak{T}_{\infty}^{0, n} \stackrel{\delta}{\longrightarrow} \mathfrak{E}_{1} \stackrel{\delta}{\longrightarrow} \cdots
$$

and the corresponding complex of differential algebras of their global sections

$$
0 \rightarrow \mathbb{R} \rightarrow \Gamma\left(\mathfrak{T}_{\infty}^{0}\right) \stackrel{d_{H}}{\longrightarrow} \Gamma\left(\mathfrak{T}_{\infty}^{0,1}\right) \stackrel{d_{H}}{\longrightarrow} \cdots \stackrel{d_{H}}{\longrightarrow} \Gamma\left(\mathfrak{T}_{\infty}^{0, n}\right) \stackrel{\delta}{\longrightarrow} \Gamma\left(\mathfrak{E}_{1}\right) \stackrel{\delta}{\longrightarrow} \cdots
$$

By virtue of the Lemma 1, the variational complex (6) is exact. The sheaves $\mathfrak{T}^{k, m}$ in this complex are sheaves of $\Gamma\left(\mathfrak{T}_{\infty}^{0}\right)$-modules and, consequently, are fine. One can prove that the sheaves $\mathfrak{E}_{k}$, being projections $\tau\left(\mathfrak{T}_{\infty}^{k, n}\right)$ of sheaves of $\Gamma\left(\mathfrak{T}_{\infty}^{0}\right)$-modules, are also fine 
[15, 16]. Consequently, the variational complex (6) is the fine resolution of the constant sheaf $\mathbb{R}$ on $J^{\infty} Y$. Then we come to the following.

Proposition 2. Cohomology of the complex (17) equals the de Rham cohomology of the fibre bundle $Y$.

Proof. By virtue of the above mentioned abstract de Rham theorem [18, there is an isomorphism between the cohomology of the complex (7) and the cohomology $H^{*}\left(J^{\infty} Y, \mathbb{R}\right)$ of the paracompact space $J^{\infty} Y$ with coefficients in the constant sheaf $\mathbb{R}$. Since $Y$ is a strong deformation retract of $J^{\infty} Y[3]$, the cohomology $H^{*}\left(J^{\infty} Y, \mathbb{R}\right)$ is isomorphic to the cohomology $H^{*}(Y, \mathbb{R})$ of $Y$ with coefficients in the constant sheaf $\mathbb{R}[12]$ and, consequently, to the de Rham cohomology $H^{*}(Y)$ of $Y$.

Proposition (2) recovers the results of [2, 27], but we also note the following. Let us consider the de Rham complex of sheaves

$$
0 \rightarrow \mathbb{R} \rightarrow \mathfrak{T}_{\infty}^{0} \stackrel{d}{\longrightarrow} \mathfrak{T}_{\infty}^{1} \stackrel{d}{\longrightarrow} \cdots
$$

on $J^{\infty} Y$ and the corresponding complex of differential algebras

$$
0 \rightarrow \mathbb{R} \rightarrow \Gamma\left(\mathfrak{T}_{\infty}^{0}\right) \stackrel{d}{\longrightarrow} \Gamma\left(\mathfrak{T}_{\infty}^{1}\right) \stackrel{d}{\longrightarrow} \cdots
$$

The complex (8) is exact due to the Poincaré lemma, and is a fine resolution of the constant sheaf $\mathbb{R}$ on $J^{\infty} Y$. Then, similarly to Proposition 2, we obtain that the de Rham cohomology of the differential algebra $\Gamma\left(\mathfrak{T}_{\infty}^{*}\right)$ is isomorphic to that $H^{*}(Y)$ of the fibre bundle $Y$. It follows that every closed form $\phi \in \Gamma\left(\mathfrak{T}_{\infty}^{*}\right)$ is split into the sum

$$
\sigma=\varphi+d \xi, \quad \xi \in \Gamma\left(\mathfrak{T}_{\infty}^{*}\right)
$$

where $\varphi$ is a closed form on the fibre bundle $Y$.

The relation (4) for $\tau$ and the relation $h_{0} d=d_{H} h_{0}$ for $h_{0}$ define a homomorphisms of the de Rham complex (9) of the algebra $\Gamma\left(\mathfrak{T}_{\infty}^{*}\right)$ to its variational complex (7), and the corresponding homomorphism of their cohomology groups is an isomorphism. Then, the splitting (10) leads to the following decompositions.

Proposition 3. Any $d_{H}$-closed form $\sigma \in \Gamma\left(\mathcal{T}^{0, m}\right), m<n$, is represented by the sum

$$
\sigma=h_{0} \varphi+d_{H} \xi, \quad \xi \in \Gamma\left(\mathfrak{T}_{\infty}^{0, m-1}\right)
$$

where $\varphi$ is a closed $m$-form on $Y$. Any $\delta$-closed form $\sigma \in \Gamma\left(\mathfrak{T}^{k, n}\right), k \geq 0$, is split into

$$
\begin{array}{rlrl}
\sigma & =h_{0} \varphi+d_{H} \xi, & k=0, & \xi \in \Gamma\left(\mathcal{T}_{\infty}^{0, n-1}\right), \\
\sigma=\tau(\varphi)+\delta(\xi), & k=1, & \xi \in \Gamma\left(\mathcal{T}_{\infty}^{0, n}\right), \\
\sigma=\tau(\varphi)+\delta(\xi), & k>1, & \xi \in \Gamma\left(\mathfrak{E}_{k-1}\right),
\end{array}
$$


where $\varphi$ is a closed $n+k$-form on $Y$.

Let us now return to the differential algebra $\mathcal{O}_{\infty}^{*}$. The following is proved [15, 16].

Proposition 4. The differential algebra $\mathcal{O}_{\infty}^{*}$ has the same $d_{-}, d_{H^{-}}$and $\delta$-cohomology as $\Gamma\left(\mathfrak{T}_{\infty}^{*}\right)$.

It follows that cohomology of the variational complex (5) of the algebra $\mathcal{O}_{\infty}^{*}$ is equal to the de Rham cohomology of the fibre bundle $Y$. Furthermore, if $\sigma$ in decompositions (11) - (14) is an element of $\mathcal{O}_{\infty}^{*} \subset \Gamma\left(\mathfrak{T}_{\infty}^{*}\right)$, then $\xi$ is so.

In quantum field theory, all physical fields are linear or affine quantities. Therefore, let $Y \rightarrow X$ is an affine bundle. Then $X$ is a strong deformation retract of $Y$ and the de Rham cohomology of $Y$ is equal to that of $X$. In this case, cohomology of the variational complex (5) equals to the de Rham cohomology of the base manifold $X$. Hence, every $d_{H^{-}}$closed form $\phi \in \mathcal{O}_{\infty}^{0, m<n}$ is split into the sum

$$
\phi=\varphi+d_{H} \xi, \quad \xi \in \mathcal{O}_{\infty}^{0, m-1}
$$

where $\varphi$ is a closed form on $X$. Any $\delta$-closed form $\sigma \in \mathcal{O}^{0, n}$ is split into

$$
\sigma=\varphi+d_{H} \xi, \quad \xi \in \mathcal{O}_{\infty}^{0, n-1}
$$

where $\varphi$ is a non-exact $n$-form on $X$.

\section{$3 \quad$ Differential geometry of ghosts}

Different geometric models of odd ghosts have been suggested. For instance, a ghost field in the Yang-Mills theory on a principal bundle has been described as the Maurer-Cartan form on the gauge group (see, e.g., [9, 26, 28]). This description however is not extended to other gauge theories and to other odd elements of the physical basis. We provide the following geometric model of odd fields on a smooth manifold $X$ [22, 25].

Let $Y \rightarrow X$ be a vector bundle with an $m$-dimensional typical fibre $V$ and $Y^{*} \rightarrow X$ the dual of $Y$. We consider the exterior bundle

$$
\wedge Y^{*}=\mathbb{R} \underset{X}{\oplus}\left(\bigoplus_{k=1}^{m} \stackrel{k}{\wedge} Y^{*}\right)
$$

whose typical fibre is the finitely generated Grassmann algebra $\wedge V^{*}$. Sections of the exterior bundle (17) are called graded functions. Let $\mathcal{A}_{Y}$ denote the sheaf of germs of graded functions on $X$. The pair $\left(X, \mathcal{A}_{Y}\right)$ is a graded manifold with the body manifold 
$X$ and the structure sheaf $\mathcal{A}_{Y}$ [6, 20]. We agree to call it a simple graded manifold with the characteristic vector bundle $Y$. Note that any graded manifold $(X, \mathcal{A})$ is isomorphic to some simple graded manifold, but this isomorphism fails to be canonical [6, [].

Given a bundle atlas $\left\{\left(U ; x^{\lambda}, y^{a}\right)\right\}$ of $Y$ with transition functions $y^{\prime a}=\rho_{b}^{a}(x) y^{b}$, let $\left\{c^{a}\right\}$ be the corresponding fibre bases for $Y^{*} \rightarrow X$, together with the transition functions $c^{\prime a}=\rho_{b}^{a}(x) c^{b}$. We will call $\left(x^{\lambda}, c^{a}\right)$ the local basis for the simple graded manifold $\left(X, \mathcal{A}_{Y}\right)$. With respect to this basis, graded functions read

$$
f=\sum_{k=0}^{m} \frac{1}{k !} f_{a_{1} \ldots a_{k}} c^{a_{1}} \cdots c^{a_{k}},
$$

where $f_{a_{1} \cdots a_{k}}$ are local smooth real functions on $U$, and we omit the symbol of the exterior product of coframes $c$.

In BRST theory, the basis elements $c^{i}$ of a simple graded manifold can describe odd ghosts. For instance, in the Yang-Mills theory on a principal bundle $P \rightarrow X$ with the structure group $G$, the above bundle $Y$ is the Lie algebra bundle $V_{G} P=V P / G$, where $V P$ denotes the vertical tangent bundle of $P$. The typical fibre of $V_{G} P$ is the right Lie algebra $\mathfrak{g}$ of the group $G$. If $X$ is a compact manifold and $G$ is a semisimple matrix Lie group, the Sobolev completion of the set of sections of $V_{G} P \rightarrow X$ is the Lie algebra of the gauge group. The typical fibre of the dual $V_{G}^{*} P$ of $V_{G} P$ is the coalgebra $\mathfrak{g}^{*}$. Let $\left\{\varepsilon_{r}\right\}$ be a basis for $\mathfrak{g},\left\{e_{r}\right\}$ the corresponding fibre bases for $V_{G} P$, and $\left\{C^{r}\right\}$ the dual coframes in $V_{G}^{*} P$. Elements $C^{r}$ of these coframes play the role of ghosts in the BRST extension of the Yang-Mills theory. Indeed, the canonical section $C=C^{r} \otimes e_{r}$ of the tensor product $V_{G}^{*} P \otimes V_{G} P$ is the above mentioned Maurer-Cartan form on the gauge group which one regards as a ghost field. In the heuristic formulation of BRST theory, $C$ plays the role of a generator of gauge transformations with odd parameters, i.e., is the BRST operator.

Let $\mathfrak{d} \mathcal{A}_{Y}$ be the sheaf of graded derivations of the sheaf $\mathcal{A}_{Y}$. Its sections are called graded vector fields on the graded manifold $\left(X, \mathcal{A}_{Y}\right)$ (or, simply, on $X$ ). Any graded vector field $u$ on an open subset $U \subset X$ is a graded derivation of the graded algebra $\Gamma\left(U, \mathcal{A}_{Y}\right)$ of local graded functions on $U$, i.e.,

$$
u\left(f f^{\prime}\right)=u(f) f^{\prime}+(-1)^{[u][f]} f u\left(f^{\prime}\right), \quad f, f^{\prime} \in \Gamma\left(U, \mathcal{A}_{Y}\right),
$$

where [.] denotes the Grassmann parity. The $\mathfrak{d} \mathcal{A}_{Y}$ is a sheaf of Lie superalgebras with respect to the bracket

$$
\left[u, u^{\prime}\right]=u u^{\prime}+(-1)^{[u]\left[u^{\prime}\right]+1} u^{\prime} u .
$$


Graded vector fields on a simple graded manifold can be seen as sections of a vector bundle as follows. Due to the canonical splitting $V Y \cong Y \times Y$, the vertical tangent bundle $V Y \rightarrow Y$ of $Y \rightarrow X$ can be provided with the fibre bases $\left\{\partial / \partial c^{a}\right\}$, dual of $\left\{c^{a}\right\}$. These are the fibre basis for $\operatorname{pr}_{2} V Y \cong Y$. Then a graded vector field on a trivialization domain $U$ reads

$$
u=u^{\lambda} \partial_{\lambda}+u^{a} \frac{\partial}{\partial c^{a}}
$$

where $u^{\lambda}, u^{a}$ are local graded functions [6, 22]. It yields a derivation of $\Gamma\left(U, \mathcal{A}_{Y}\right)$ by the rule

$$
\left.u\left(f_{a \ldots b} c^{a} \cdots c^{b}\right)=u^{\lambda} \partial_{\lambda}\left(f_{a \ldots b}\right) c^{a} \cdots c^{b}+u^{d} f_{a \ldots b} \frac{\partial}{\partial c^{d}}\right\rfloor\left(c^{a} \cdots c^{b}\right) .
$$

This rule implies the corresponding coordinate transformation law

$$
u^{\prime \lambda}=u^{\lambda}, \quad u^{\prime a}=\rho_{j}^{a} u^{j}+u^{\lambda} \partial_{\lambda}\left(\rho_{j}^{a}\right) c^{j}
$$

of graded vector fields. It follows that graded vector fields (18) can be represented by sections of the vector bundle $\mathcal{V}_{Y} \rightarrow X$ which is locally isomorphic to the vector bundle

$$
\left.\left.\mathcal{V}_{Y}\right|_{U} \approx \wedge Y^{*} \underset{X}{\otimes}\left(\operatorname{pr}_{2} V Y \underset{X}{\oplus} T X\right)\right|_{U}
$$

and has the bundle coordinates $\left(x_{a_{1} \ldots a_{k}}^{\lambda}, v_{b_{1} \ldots b_{k}}^{i}\right), k=0, \ldots, m$, together with the transition functions

$$
\begin{aligned}
& x_{i_{1} \ldots i_{k}}^{\prime \lambda}=\rho^{-1 a_{1}} \cdots \rho^{-1 a_{k}} x_{i_{k} \ldots a_{k}}^{\lambda}, \\
& v_{j_{1} \ldots j_{k}}^{i}=\rho^{-1 b_{1}} \cdots \rho_{j_{1}}^{-1 b_{k}}\left[\rho_{j}^{i} v_{b_{1} \ldots b_{k}}^{j}+\frac{k !}{(k-1) !} x_{b_{1} \ldots b_{k-1}}^{\lambda} \partial_{\lambda} \rho_{b_{k}}^{i}\right] .
\end{aligned}
$$

There is the exact sequence

$$
0 \rightarrow \wedge Y^{*} \underset{X}{\otimes} \operatorname{pr}_{2} V Y \rightarrow \mathcal{V}_{Y} \rightarrow \wedge Y^{*} \underset{X}{\otimes} T X \rightarrow 0
$$

of vector bundles over $X$. Its splitting

$$
\widetilde{\gamma}: \dot{x}^{\lambda} \partial_{\lambda} \mapsto \dot{x}^{\lambda}\left(\partial_{\lambda}+\widetilde{\gamma}_{\lambda}^{a} \frac{\partial}{\partial c^{a}}\right)
$$

transforms every vector field $\tau$ on $X$ into the graded vector field

$$
\tau=\tau^{\lambda} \partial_{\alpha} \mapsto \nabla_{\tau}=\tau^{\lambda}\left(\partial_{\lambda}+\widetilde{\gamma}_{\lambda}^{a} \frac{\partial}{\partial c^{a}}\right)
$$


which is a graded derivation of the sheaf $\mathcal{A}_{Y}$ satisfying the Leibniz rule

$$
\left.\nabla_{\tau}(s f)=(\tau\rfloor d s\right) f+s \nabla_{\tau}(f), \quad f \in \Gamma\left(U, \mathcal{A}_{Y}\right), \quad s \in C^{\infty}(X),
$$

for any open subset $U \subset X$. Therefore, one can think of the splitting (201) as being a graded connection on the simple graded manifold $\left(X, \mathcal{A}_{Y}\right)$ [22]. It should be emphasized that this notion of a graded connection differs from that of a connection on a graded fibre bundle in [1]. In particular, every linear connection

$$
\gamma=d x^{\lambda} \otimes\left(\partial_{\lambda}+\gamma_{\lambda}^{a} b v^{b} \partial_{a}\right)
$$

on the vector bundle $Y \rightarrow X$ yields the graded connection

$$
\widetilde{\gamma}=d x^{\lambda} \otimes\left(\partial_{\lambda}+\gamma_{\lambda}{ }^{a}{ }_{b} c^{b} \frac{\partial}{\partial c^{a}}\right) .
$$

For instance, let $Y$ be the Lie algebra bundle $V_{G} P$ in the Yang-Mills theory on a $G$-principal bundle $P$. Every principal connection $A$ on $P \rightarrow X$ yields a linear connection

$$
A=d x^{\lambda} \otimes\left(\partial_{\lambda}-c_{p q}^{r} A_{\lambda}^{p} \xi^{q} e_{r}\right)
$$

on $V_{G} P \rightarrow X$ [22] and, consequently, the graded connection on ghosts

$$
\widetilde{A}=d x^{\lambda} \otimes\left(\partial_{\lambda}-c_{p q}^{r} A_{\lambda}^{p} C^{q} \frac{\partial}{C^{r}}\right),
$$

where $c_{p q}^{r}$ are the structure constants of the Lie algebra $\mathfrak{g}$.

Let $\mathcal{V}_{Y}^{*} \rightarrow X$ be a vector bundle which is the pointwise $\wedge Y^{*}$-dual of $\mathcal{V}_{Y}$. It is locally isomorphic to the vector bundle

$$
\left.\left.\mathcal{V}_{Y}^{*}\right|_{U} \approx \wedge Y^{*} \underset{X}{\otimes}\left(\operatorname{pr}_{2} V Y^{*} \underset{X}{\oplus} T^{*} X\right)\right|_{U} .
$$

With respect to the dual bases $\left\{d x^{\lambda}\right\}$ for $T^{*} X$ and $\left\{d c^{b}\right\}$ for $\operatorname{pr}_{2} V^{*} Y=Y^{*}$, sections of the vector bundle $\mathcal{V}_{Y}^{*}$ take the coordinate form

$$
\phi=\phi_{\lambda} d x^{\lambda}+\phi_{a} d c^{a},
$$

together with transition functions

$$
\phi_{a}^{\prime}=\rho_{a}^{-1 b} \phi_{b}, \quad \phi_{\lambda}^{\prime}=\phi_{\lambda}+\rho_{a}^{-1 b} \partial_{\lambda}\left(\rho_{j}^{a}\right) \phi_{b} c^{j} .
$$

They are treated as graded 1-forms on the graded manifold $\left(X, \mathcal{A}_{Y}\right)$. 
The sheaf $\mathfrak{O}^{1} \mathcal{A}_{Y}$ of germs of sections of the vector bundle $\mathcal{V}_{Y}^{*} \rightarrow X$ is the dual of the sheaf $\mathfrak{d} \mathcal{A}_{Y}$, where the duality morphism is given by the interior product

$$
u\rfloor \phi=u^{\lambda} \phi_{\lambda}+(-1)^{\left[\phi_{a}\right]} u^{a} \phi_{a} .
$$

Graded $k$-forms $\phi$ are defined as sections of the graded exterior bundle $\stackrel{\wedge}{X}_{Y}^{k} \mathcal{V}_{Y}^{*}$ such that

$$
\phi \wedge \sigma=(-1)^{|\phi||\sigma|+[\phi][\sigma]} \sigma \wedge \phi
$$

where |.| denotes the form degree. The graded exterior differential $d$ of graded functions is introduced in accordance with the condition $u\rfloor d f=u(f)$ for an arbitrary graded vector field $u$, and is extended uniquely graded exterior forms by the rules

$$
d(\phi \wedge \sigma)=(d \phi) \wedge \sigma+(-1)^{|\phi|} \phi \wedge(d \sigma), \quad d \circ d=0
$$

It takes the coordinate form

$$
d \phi=d x^{\lambda} \wedge \partial_{\lambda}(\phi)+d c^{a} \wedge \frac{\partial}{\partial c^{a}}(\phi)
$$

where the left derivatives $\partial_{\lambda}, \partial / \partial c^{a}$ act on coefficients of graded exterior forms by the rule (19), and they are graded commutative with the forms $d x^{\lambda}, d c^{a}$.

With $d$, graded exterior forms constitute a graded differential algebra $\mathcal{O}^{*} \mathcal{A}_{Y}$, where $\mathcal{O}^{0} \mathcal{A}_{Y}=\Gamma\left(\mathcal{A}_{Y}\right)$ is the graded commutative ring of graded functions on $X$. There is a monomorphism of differential algebras $\mathcal{O}^{*}(X) \rightarrow \mathcal{O}^{*} \mathcal{A}_{Y}$. Let $\mathfrak{T}^{*} \mathcal{A}_{Y}$ denote the sheaf of germs of graded exterior forms on $X$. Then $\mathcal{O}^{*} \mathcal{A}_{Y}=\Gamma\left(\mathfrak{T}^{*} \mathcal{A}_{Y}\right)$.

If the basis elements $c^{a}$ of the graded manifold $\left(X, \mathcal{A}_{Y}\right)$ are treated as ghosts of ghost number 1 , graded exterior forms $\phi \in \mathcal{O}^{*} \mathcal{A}_{Y}$ can also be provided with a ghost number by the rule

$$
\operatorname{gh}\left(d c^{a}\right)=1, \quad \operatorname{gh}\left(d x^{\lambda}\right)=0 .
$$

Then the Grassmann parity $[\phi]$ is equal to $\operatorname{gh}(\phi) \bmod 2$. Ona also introduces the total ghost number $\operatorname{gh}(\phi)+|\phi|$.

\section{Jets of ghosts}

As was mentioned above, the antibracket and the BRST opreator in the field-antifield BRST theory of [4, 5, 11] are expressed in terms of jets of ghosts. For example, the BRST transformation of gauge potentials $a_{\lambda}^{m}$ in the Yang-Mills theory reads

$$
\mathbf{s} a_{\lambda}^{r}=C_{\lambda}^{r}+c_{p q}^{r} a_{\lambda}^{p} C^{q}
$$


where $C_{\lambda}^{r}$ are jets of ghosts $C^{r}$ introduced usually in a heuristic way. We will describe jets of odd fields as elements of a particular simple graded manifold.

Let $Y \rightarrow X$ be the characteristic vector bundle of a simple graded manifold $\left(X, \mathcal{A}_{Y}\right)$. The $r$-order jet manifold $J^{r} Y$ of $Y$ is also a vector bundle over $X$. Let us consider the simple graded manifold $\left(X, \mathcal{A}_{J^{r} Y}\right)$ with the characteristic vector bundle $J^{r} Y \rightarrow X$. Its local basis is $\left\{x^{\lambda}, c_{\Lambda}^{a}\right\}, 0 \leq|\Lambda| \leq r$, together with the transition functions

$$
c_{\lambda+\Lambda}^{\prime a}=d_{\lambda}\left(\rho_{j}^{a} c_{\Lambda}^{j}\right),
$$

where

$$
d_{\lambda}=\partial_{\lambda}+\sum_{|\Lambda|<r} c_{\lambda+\Lambda}^{a} \frac{\partial}{\partial c_{\Lambda}^{a}}
$$

denotes the graded total derivative. In view of the transition functions (23), one can think of $\left(X, \mathcal{A}_{J^{r} Y}\right)$ as being a graded $r$-order jet manifold of the graded manifold $\left(X, \mathcal{A}_{Y}\right)$. It should be emphasized that this notion differs from that of a graded jet manifold of a graded fibre bundle 24].

Let $\mathcal{O}^{*} \mathcal{A}_{J^{r} Y}$ be the differential algebra of graded exterior forms on the simple graded manifold $\left(X, \mathcal{A}_{J^{r} Y}\right)$. Being a linear bundle morphism of vector bundles over $X$, the affine bundle $\pi_{r-1}^{r}: J^{r} Y \rightarrow J^{r-1} Y$ yields the corresponding morphism of simple graded manifolds $\left(X, \mathcal{A}_{J^{r} Y}\right) \rightarrow\left(X, \mathcal{A}_{J^{r-1} Y}\right)$ [22] and the pull-back monomorphism of differential algebras $\mathcal{O}^{*} \mathcal{A}_{J^{r-1} Y} \rightarrow \mathcal{O}^{*} \mathcal{A}_{J^{r} Y}$. With the inverse system of jet manifolds (1), we have the direct system of differential algebras

$$
\mathcal{O}^{*} \mathcal{A}_{Y} \longrightarrow \mathcal{O}^{*} \mathcal{A}_{J^{1} Y} \longrightarrow \cdots \mathcal{O}^{*} \mathcal{A}_{J^{r} Y} \stackrel{\pi_{r}^{r+1 *}}{\longrightarrow} \cdots
$$

Its direct limit $\mathcal{O}_{\infty}^{*} \mathcal{A}_{Y}$ consists of graded exterior forms on graded jet manifolds $\left(X, \mathcal{A}_{J^{r} Y}\right)$, $0 \leq r$, modulo the pull-back identification. It is a locally free $C^{\infty}(X)$-algebra generated by the elements

$$
\left(1, d x^{\lambda}, c_{\Lambda}^{a}, \theta_{\Lambda}^{a}=d c_{\Lambda}^{a}-c_{\lambda+\Lambda}^{a} d x^{\lambda}\right), \quad 0 \leq|\Lambda| .
$$

We have the corresponding decomposition of $\mathcal{O}_{\infty}^{*} \mathcal{A}_{Y}$ into $\mathcal{O}_{\infty}^{0} \mathcal{A}_{Y}$-modules $\mathcal{O}_{\infty}^{k, s} \mathcal{A}_{Y}$ of $k$ contact and $s$-horizontal graded forms. Accordingly, the graded exterior differential $d$ on the algebra $\mathcal{O}_{\infty}^{*} \mathcal{A}_{Y}$ is split into the sum $d=d_{H}+d_{V}$ of the graded horizontal differential

$$
d_{H}(\phi)=d x^{\lambda} \wedge d_{\lambda}(\phi), \quad \phi \in \mathcal{O}^{*} \mathcal{A}_{\infty}
$$

and the graded vertical differential $d_{V}$.

If the basis elements $c^{a}$ of the graded manifold $\left(X, \mathcal{A}_{Y}\right)$ are treated as ghosts of ghost number 1 , jets of ghosts $c_{\Lambda}^{a}$ and the graded exterior forms $d c_{\Lambda}^{a}$ are also provided with ghost number 1 . 


\section{$5 \quad$ Even fields and antifields}

In order to describe odd and even elements of the physical basis of BRST theory on the same footing, let us generalize the notion of a graded manifold to graded commutative algebras generated both by odd and even elements [21].

Let $Y=Y_{0} \oplus Y_{1}$ be the Whitney sum of vector bundles $Y_{0} \rightarrow X$ and $Y_{1} \rightarrow X$. We treat it as a bundle of graded vector spaces with the typical fibre $V=V_{0} \oplus V_{1}$. Let us consider the quotient of the tensor bundle

$$
\otimes Y^{*}=\underset{k=0}{\oplus}\left(\stackrel{k}{\otimes} Y^{*}\right)
$$

by the elements

$$
y_{0} y_{0}^{\prime}-y_{0}^{\prime} y_{0}, \quad y_{1} y_{1}^{\prime}+y_{1}^{\prime} y_{1}, \quad y_{0} y_{1}-y_{1} y_{0}
$$

for all $y_{0}, y_{0}^{\prime} \in Y_{0 x}^{*}, y_{1}, y_{1}^{\prime} \in Y_{1 x}^{*}$, and $x \in X$. This is an infinite-dimensional vector bundle which we will denote by $\wedge Y^{*}$. Global sections of $\wedge Y^{*}$ constitute a graded commutative algebra $\mathcal{A}_{Y}(X)$ which is the product over $C^{\infty}(X)$ of the commutative algebra $\mathcal{A}_{0}(X)$ of global sections of the symmetric bundle $\vee Y_{0}^{*} \rightarrow X$ and the graded algebra $\mathcal{A}_{1}(X)$ of global sections of the exterior bundle $\wedge Y_{1}^{*} \rightarrow X$.

Let $\mathcal{A}_{Y}, \mathcal{A}_{0}$ and $\mathcal{A}_{1}$ be the sheaves of germs of sections of the vector bundles $\wedge Y^{*}$, $\vee Y_{0}^{*}$ and $\wedge Y_{1}^{*}$, respectively. For instance, the pair $\left(X, \mathcal{A}_{1}\right)$ is a familiar simple graded manifold. For the sake of brevity, we therefore agree to call $\left(X, \mathcal{A}_{Y}\right)$ the graded commutative manifold with the characteristic vector bundle $Y$. Given a coordinate chart $\left(x^{\lambda}, y_{0}^{i}, y_{1}^{a}\right)$ of $Y$, the local basis for $\left(X, \mathcal{A}_{Y}\right)$ is $\left(x^{\lambda}, c_{0}^{i}, c_{1}^{a}\right)$, where $\left\{c_{0}^{i}\right\}$ and $\left\{c_{1}^{a}\right\}$ are the fibre bases for the vector bundles $Y_{0}^{*}$ and $Y_{1}^{*}$, respectively. Then a straightforward repetition of all the above constructions for a simple graded manifold provides us with the differential algebra $\mathcal{O}^{*} \mathcal{A}_{\infty}$ of graded commutative exterior forms on $X$. This is a $C^{\infty}(X)$-algebra generated locally by the elements $\left(1, c_{0 \Lambda}^{i}, c_{1 \Lambda}^{a}, d x^{\lambda}, \theta_{0 \Lambda}^{i}, \theta_{1 \Lambda}^{a}\right), 0 \leq|\Lambda|$. Its $C^{\infty}(X)$-subalgebra $\mathcal{O}^{*} \mathcal{A}_{1 \infty}$, generated locally by the elements $\left(1, c_{1 \Lambda}^{i}, d x^{\lambda}, \theta_{1 \Lambda}^{i}\right)$, is exactly the differential algebra $\mathcal{O}_{\infty}^{*} \mathcal{A}_{Y_{1}}$ on the simple graded manifold $\left(X, \mathcal{A}_{1}\right)$. The $C^{\infty}(X)$-subalgebra $\mathcal{O}^{*} \mathcal{A}_{0 \infty}$ of $\mathcal{O}^{*} \mathcal{A}_{\infty}$, generated locally by the elements $\left(1, c_{0 \Lambda}^{i}, d x^{\lambda}, \theta_{0 \Lambda}^{i}\right), 0 \leq|\Lambda|$, is isomorphic to the polynomial subalgebra of the differential algebra $\mathcal{O}_{\infty}^{*}$ of exterior forms on the infinite order jet manifold $J^{\infty} Y_{0}$ of the vector bundle $Y_{0} \rightarrow X$. This isomorphism is performed by the formal assignment $y_{0 \Lambda}^{i} \leftrightarrow c_{0 \Lambda}^{i}$ which is preserved by the transition functions (2) and (23).

In the field-antifield BRST theory, the basis elements $c_{0 \Lambda}^{i}$ of the algebra $\mathcal{O}^{*} \mathcal{A}_{\infty}$ can characterize even elements of the physical basis and their jets, while $c_{1 \Lambda}^{a}$ describe odd elements of the physical basis and their jets. 
It should be emphasized that, in the jet formulation of the field-antifield BRST theory, antifields can be introduced on the same footing as physical fields and ghosts. Let us denote physical fields and ghosts by the collective symbol $\Phi^{A}$. Let $E$ be the characteristic vector bundle of the graded commutative manifold generated by $\Phi^{A}$. Treated as source coefficients of BRST transformations, antifields $\Phi_{A}^{*}$ are represented by elements of the graded commutative manifold whose structure vector bundle is $\wedge^{n} T^{*} X \otimes E^{*}$ (cf. the geometric treatment of antifields in functional BRST formalism [19, 30]).

In particular, gauge potentials in the Yang-Mills theory are represented by sections of the affine bundle $J^{1} P / G \rightarrow X$ modelled on the vector bundle $T X \otimes V_{G}^{*} P$. Their antifields are the basis elements of the vector bundle $\wedge^{n} T^{*} X \otimes T^{*} X \otimes V_{G} P$. Accordingly, the antifields of ghosts in the Yang-Mills theory are the basis elements of the vector bundle $\wedge^{n} T^{*} X \otimes V_{G}^{*} P$.

\section{The variational complex in BRST theory}

The differential algebra $\mathcal{O}^{*} \mathcal{A}_{\infty}$ gives everything that one needs for a global formulation of the Lagrangian field-antifield BRST theory in jet terms. In particular, let us consider the short variational complex

$$
0 \longrightarrow \mathbb{R} \longrightarrow \mathcal{O}^{0} \mathcal{A}_{\infty} \stackrel{d_{H}}{\longrightarrow} \mathcal{O}^{0,1} \mathcal{A}_{\infty} \stackrel{d_{H}}{\longrightarrow} \cdots \stackrel{d_{H}}{\longrightarrow} \mathcal{O}^{0, n} \mathcal{A}_{\infty} \stackrel{\delta}{\longrightarrow} \operatorname{Im} \delta \rightarrow 0,
$$

where $\delta$ is given by the expression

$$
\delta(L)=(-1)^{|\Lambda|} \theta^{a} \wedge d_{\Lambda}\left(\partial_{a}^{\Lambda} L\right), \quad L \in \mathcal{O}^{0, n} \mathcal{A}_{\infty},
$$

with respect to a physical basis $\left\{\zeta^{a}\right\}$. The variational complex (24) provides the algebraic approach to the antibracket technique, where one can think of elements of $\mathcal{O}^{0, n} \mathcal{A}_{\infty}$ as being Lagrangians of fields, ghosts and antifields.

To obtain cohomology of the variational complex (24), one can follow exactly the procedure in Section 2. Let us consider the sheaf $\mathfrak{T}^{*} \mathcal{A}_{\infty}$ of germs of graded commutative exterior forms $\phi \in \mathcal{O}^{*} \mathcal{A}_{\infty}$ and the differential algebra $\Gamma\left(\mathfrak{T}^{*} \mathcal{A}_{\infty}\right)$ of global sections of this sheaf. We have the short variational complex of sheaves

$$
0 \longrightarrow \mathbb{R} \longrightarrow \mathfrak{T}^{0} \mathcal{A}_{\infty} \stackrel{d_{H}}{\longrightarrow} \mathfrak{T}^{0,1} \mathcal{A}_{\infty} \stackrel{d_{H}}{\longrightarrow} \cdots \stackrel{d_{H}}{\longrightarrow} \mathfrak{T}^{0, n} \mathcal{A}_{\infty} \stackrel{\delta}{\longrightarrow} \operatorname{Im} \delta \rightarrow 0 .
$$

There is the following variant of the algebraic Poincaré lemma [10, 11, 13].

Lemma 5. The complex (25) is exact. 
Since $\mathfrak{T}^{0, *} \mathcal{A}_{\infty}$ are sheaves of $C^{\infty}(X)$-modules, they are fine and acyclic. Without studying the acyclicity of the sheaf $\operatorname{Im} \delta$, we can apply a minor modification of the abstract de Rham theorem [15, 27] to the complex (25), and obtain the following.

Proposition 6. Cohomology of the complex

$$
0 \longrightarrow \mathbb{R} \longrightarrow \Gamma\left(\mathfrak{T}^{0} \mathcal{A}_{\infty}\right) \stackrel{d_{H}}{\longrightarrow} \Gamma\left(\mathfrak{T}^{0,1} \mathcal{A}_{\infty}\right) \stackrel{d_{H}}{\longrightarrow} \cdots \stackrel{d_{H}}{\longrightarrow} \Gamma\left(\mathfrak{T}^{0, n} \mathcal{A}_{\infty}\right) \stackrel{\delta}{\longrightarrow} \operatorname{Im} \delta \rightarrow 0
$$

is isomorphic to the de Rham cohomology of $X$.

This cohomology isomorphism is performed by a monomorphism of the de Rham complex of exterior forms on $X$ to the complex (26); that leads to the following.

Corollary 7. Every $d_{H}$-closed form $\phi \in \Gamma\left(\mathfrak{T}^{0, m<n} \mathcal{A}_{\infty}\right)$ is split into the sum

$$
\phi=\varphi+d_{H} \xi, \quad \xi \in \Gamma\left(\mathfrak{T}^{0, m-1} \mathcal{A}_{\infty}\right),
$$

where $\varphi$ is a closed $m$-form on $X$. Every $\delta$-closed form $\phi \in \Gamma\left(\mathfrak{T}^{0, n} \mathcal{A}_{\infty}\right)$ is split into the sum

$$
\phi=\varphi+d_{H} \xi, \quad \xi \in \Gamma\left(\mathfrak{T}^{0, n-1} \mathcal{A}_{\infty}\right),
$$

where $\varphi$ is a non-exact $n$-form on $X$.

Turn now to the short variational complex (24). Its cohomology is equal to that of the complex (26). The proof of this fact is a repetition of that of Proposition 1 where exterior forms on $J^{\infty} Y$ are replaced with graded commutative forms on $X$ and, accordingly, Lemma 5 and Corollary 0 are quoted. It follows that a graded commutative form exterior $\xi$ in the expressions (27) and (28) belongs to the algebra $\mathcal{O}^{*} \mathcal{A}_{\infty}$ whenever $\phi$ does.

We also mention the important case of a BRST theory where Lagrangians are independent on coordinates $x^{\lambda}$. Let us consider the subsheaf $\widetilde{\mathfrak{T}}^{*} \mathcal{A}_{\infty}$ of the sheaf $\mathfrak{T}^{*} \mathcal{A}_{\infty}$ which consists of germs of $x$-independent graded commutative exterior forms. Then we have the subcomplex

$$
0 \longrightarrow \mathbb{R} \longrightarrow \overline{\mathfrak{T}}^{0} \mathcal{A}_{\infty} \stackrel{d_{H}}{\longrightarrow} \overline{\mathfrak{T}}^{0,1} \mathcal{A}_{\infty} \stackrel{d_{H}}{\longrightarrow} \cdots \stackrel{d_{H}}{\longrightarrow} \overline{\mathfrak{T}}^{0, n} \mathcal{A}_{\infty} \stackrel{\delta}{\longrightarrow} \operatorname{Im} \delta \rightarrow 0
$$

of the complex (25) and the corresponding subcomplex

$$
0 \longrightarrow \mathbb{R} \longrightarrow \Gamma\left(\overline{\mathfrak{T}}^{0} \mathcal{A}_{\infty}\right) \stackrel{d_{H}}{\longrightarrow} \Gamma\left(\overline{\mathfrak{T}}^{0,1} \mathcal{A}_{\infty}\right) \stackrel{d_{H}}{\longrightarrow} \cdots \stackrel{d_{H}}{\longrightarrow} \Gamma\left(\overline{\mathfrak{T}}^{0, n} \mathcal{A}_{\infty}\right) \stackrel{\delta}{\longrightarrow} \operatorname{Im} \delta \rightarrow 0
$$

of the complex (26) which consists of $x$-independent graded commutative exterior forms. It is readily observed that these forms are of bounded jet order and $\Gamma\left(\overline{\mathfrak{T}}^{0, *} \mathcal{A}_{\infty}\right) \subset \mathcal{O}^{0, *} \mathcal{A}_{\infty}$, i.e., the complex (30) is also a subcomplex of the short variational complex (24). 
The key point is that the complex of sheaves (29) fails to be exact. The obstruction to its exactness at the term $\overline{\mathfrak{T}}_{\infty}^{0, k}$ is provided by the germs of $k$-forms on $X$ with constant coefficients [5]. Let us denote the sheaf of such germs by $S_{X}^{k}$. We have the short exact sequences of sheaves

$$
\begin{aligned}
& 0 \rightarrow \operatorname{Im} d_{H} \rightarrow \operatorname{Ker} d_{H} \rightarrow S_{X}^{k} \rightarrow 0, \quad 0<k<n, \\
& 0 \rightarrow \operatorname{Im} d_{H} \rightarrow \operatorname{Ker} \delta \rightarrow S_{X}^{n} \rightarrow 0
\end{aligned}
$$

and the corresponding sequences of modules of their global sections

$$
\begin{aligned}
& 0 \rightarrow \Gamma\left(\operatorname{Im} d_{H}\right) \rightarrow \Gamma\left(\operatorname{Ker} d_{H}\right) \rightarrow \Gamma\left(S_{X}^{k}\right) \rightarrow 0, \quad 0<k<n, \\
& 0 \rightarrow \Gamma\left(\operatorname{Im} d_{H}\right) \rightarrow \Gamma(\operatorname{Ker} \delta) \rightarrow \Gamma\left(S_{X}^{n}\right) \rightarrow 0
\end{aligned}
$$

which are exact because $S_{X}^{k<n}$ and $S_{X}^{n}$ are subsheaves of $\mathbb{R}$-modules of the sheaves Ker $d_{H}$ and $\operatorname{Ker} \delta$, respectively. Therefore, the $k$ th cohomology group of the complex (30) is isomorphic to the $\mathbb{R}$-module $\Gamma\left(S_{X}^{k}\right)$ of global constant $k$-forms, $0<k \leq n$, on the manifold $X$. Thus, any $d_{H}$-closed graded commutative $k$-form, $0<k<n$, and any $\delta$-closed graded commutative $n$-form $\phi$ are split into the sum $\phi=\varphi+d_{H} \xi$ where $\varphi \in \Gamma\left(S_{X}^{k}\right)$ and $\xi \in \Gamma\left(\mathfrak{T}^{0, k-1} \mathcal{A}_{\infty}\right)$.

Thus, we observe that the obstruction to the exactness of the variational complex in the field-antifield BRST theory on an arbitrary manifold $X$ lies only in exterior forms on $X$. In particular, it follows that the topological ambiguity of a proper solution of the master equation in the Lagrangian BRST theory reduces to exterior forms on $X$.

\section{References}

[1] A.Almorox, Supergauge theories in graded manifolds, In Differential Geometric Methods in Mathematical Physics, Lect. Notes in Mathematics, 1251, (Springer, Berlin, 1987), pp.114-136.

[2] I.Anderson and T.Duchamp, On the existence of global variational principles, Amer. J. Math. 102 (1980) 781.

[3] I.Anderson, Introduction to the variational bicomplex, Contemp. Math. 132 (1992) 51.

[4] G.Barnish, F.Brandt and M.Henneaux, Local BRST cohomology in the antifield formalism. 1. General theorems, Commun. Math. Phys. 174 (1995) 57. 
[5] G.Barnish, F.Brandt and M.Henneaux, Local BRST cohomology in gauge theories, Phys. Rep. 338 (2000) 439; E-print arXiv: hep-th/0002245.

[6] C.Bartocci, U.Bruzzo and D.Hernández Ruipérez, The Geometry of Supermanifolds (Kluwer Academic Publ., Dordrecht, 1991).

[7] M.Batchelor, The structure of supermanifolds, Trans. Amer. Math. Soc. 253 (1979) 329 .

[8] M.Bauderon, Differential geometry and Lagrangian formalism in the calculus of variations, In Differential Geometry, Calculus of Variations, and their Applications, Lecture Notes in Pure and Applied Mathematics, 100, (Marcel Dekker Inc., N.Y., 1985), pp.67-82.

[9] L.Bonora and P.Cotta-Ramusino, Some remarks on BRS transformations, anomalies and the cohomology of the Lie algebra of the group of gauge transformations, Commun. Math. Phys. 87 (1983) 589.

[10] F.Brandt, N.Dragon and M.Kreuzer, Completeness and nontriviality of the solutions of consistency conditions, Nucl. Phys. B332 (1990) 224.

[11] F.Brandt, Local BRST cohomology and covariance, Commun. Math. Phys. 190 (1997) 459 .

[12] G.Bredon, Sheaf Theory (McGraw-Hill Book Company, N.Y., 1967).

[13] N.Dragon, E-print arXiv: hep-th/9602163.

[14] G.Giachetta, L.Mangiarotti and G.Sardanashvily, New Lagrangian and Hamiltonian Methods in Field Theory (World Scientific, Singapore, 1997).

[15] G.Giachetta, L.Mangiarotti and G.Sardanashvily, E-print arXiv: mathph/0005010, math.DG/0006074.

[16] G.Giachetta, L.Mangiarotti and G.Sardanashvily, Iterated BRST cohomology, Lett. Math. Phys. 53 (2000) 143; E-print arXiv: hep-th/0006143.

[17] J.Gomis, J.Paris, S.Samuel, Antibracket, antifields and gauge theory quantization, Phys. Rep. 259 (1995) 1.

[18] F.Hirzebruch, Topological Methods in Algebraic Geometry (Springer, Berlin, 1966). 
[19] O.Khudaverdian, Geometry of superspace with even and odd brackets, J. Math. Phys. 32 (1991) 1934.

[20] B.Kostant, Graded manifolds, graded Lie theory, and prequantization, In Differential Geometric Methods in Mathematical Physics, Lect. Notes in Mathematics, 570 (Springer-Verlag, Berlin, 1977) pp. 177-306.

[21] L.Mangiarotti and G.Sardanashvily, The Koszul-Tate cohomology in covariant Hamiltonian formalism, Mod. Phys. Lett. A 14 (1999) 2201; E-print arXiv: hepth/9906001.

[22] L.Mangiarotti and G.Sardanashvily, Connections in Classical and Quantum Field Theory (World Scientific, Singapore, 2000).

[23] P.Olver, Applications of Lie Groups to Differential Equations (Springer, Berlin, 1997).

[24] D.Ruipérez and J.Masqué, Global variational calculus on graded manifolds, J. Math. Pures et Appl. 63 (1984) 283.

[25] G.Sardanashvily, SUSY-extended field theory, Int. J. Mod. Phys. A 15 (2000) 3095; E-print arXiv: hep-th/9911108.

[26] R.Schmid, Local cohomology in gauge theories, BRST transformations and anomalies, Diff. Geom. Appl. 4 (1994) 107.

[27] F.Takens, A global version of the inverse problem of the calculus of variations, $J$. Diff. Geom. 14 (1979) 543.

[28] J.Thierry-Mieg, Geometrical reinterpretation of Faddeev-Popov ghost particles and BRS transformations, J. Math. Phys. 21 (1980) 2834.

[29] W.Tulczyjew, The Euler-Lagrange resolution, In Differential Geometric Methods in Mathematical Physics, Lect. Notes in Mathematics 836 (Springer, Berlin, 1980), pp 22-48.

[30] E.Witten, A note on the antibracket formalism, Mod. Phys. Lett. A A (1990) 487. 